EXTENDED REPORT

\title{
Tibial cartilage volume change in healthy postmenopausal women: a longitudinal study
}

\author{
A E Wluka, R Wolfe, S R Davis, S Stuckey, F M Cicuttini
}

Ann Rheum Dis 2004;63:444-449. doi: 10.1136/ard.2003.008433

See end of article for authors' affiliations

...............

Correspondence to: Associated Professor Flavia Cicuttini,

Department of

Epidemiology and

Preventive Medicine,

Alfred Hospital,

Melbourne Victoria 3004

Australia; flavia.cicuttini@

med.monash.edu.au

Accepted 8 July 2003
Objective: To determine whether the amount of joint cartilage in healthy postmenopausal women is stable or changes over time, and whether oestrogen replacement therapy (ERT) influences this.

Design: A cohort study in healthy postmenopausal women without knee pain, initially selected on the basis of having either used ERT long term (more than five years) or never having used ERT.

Methods: 81 women (42 taking ERT and 39 non-users) had baseline knee radiographs and magnetic resonance imaging (MRI) on the dominant knee; 57 of these (70\%) were followed with repeat MRI approximately 2.5 years later. Knee cartilage volume was measured at baseline and at follow up. Risk factors assessed at baseline, including ERT use, were tested for their association with change in knee cartilage volume over time.

Results: 29 subjects who were initially taking ERT and 28 non-users at baseline completed the study. Total tibial articular cartilage decreased, on average, by (mean (SD)) 2.4 (3.2)\% per year (95\% confidence interval for mean, $1.5 \%$ to $3.2 \%)$. Average annual reduction in medial and lateral tibial cartilage was 2.4 $(3.6) \%(1.4 \%$ to $3.3 \%)$ and $2.3(4.2) \%(1.2 \%$ to $3.4 \%)$, respectively. No association between ERT and the rate of reduction in cartilage volume was shown.

Conclusions: Mean tibial cartilage volume loss in healthy postmenopausal women is between $1.5 \%$ and $3.2 \%$ a year. Whether this rate of change is similar throughout adult life or in men will require further investigation.
O teoarthritis is a disease of joints involving both cartilage and bone. With increasing disease severity, articular cartilage is lost. However, although much is known about what happens after disease onset at the level of the joint, little is known about initiating factors, the predisease state, and what occurs in healthy humans. Factors influencing the incidence of osteoarthritis have been identified through epidemiological and small group studies. These factors include sex (women, especially after entering the menopause), obesity, inheritance, knee injury, bending, the presence of hand osteoarthritis (Heberden's nodes), and quadriceps strength. ${ }^{12}$ There is also some evidence to suggest that oestrogen replacement therapy (ERT) may be protective against the development of osteoarthritis, although this remains controversial. ${ }^{3}$ However, how these factors affect the previously healthy joint at the level of the articular cartilage to influence the development of osteoarthritis has not been studied.

Normal age related change in joint cartilage must be defined before the effect of these factors can be clarified. The biochemical, structural, and mechanical properties of articular knee cartilage change with increasing age. Biochemical and structural changes are well described in aging cartilage. ${ }^{4-8}$ It has been proposed that these underlie the differences in mechanical properties of healthy cartilage between young and older subjects. ${ }^{8}{ }^{9}$ This study also suggested that knee articular cartilage is thinner in older subjects aged 50 to 78 years than in younger subjects aged 20 to 30 years. ${ }^{9}$ These data suggest that cartilage volume is lost in healthy adults.

In contrast to the well described changes that occur in bone with age, minimal information is available on joint cartilage. It is unknown when cartilage growth and accumulation ceases and degeneration begins. In children, increased weight bearing activity and a normal body mass index (BMI) may promote cartilage accrual. ${ }^{10}$ Recently, we have shown that subjects with osteoarthritis lose tibial cartilage at a rate of approximately 5\% a year. ${ }^{11}$ However, there are limited longitudinal data on the rate of change in normal healthy adults.

We examined a cohort of healthy postmenopausal women with no symptoms of knee osteoarthritis to determine the change in tibial cartilage over two years and to examine factors that may affect change, including ERT.

\section{METHODS}

Postmenopausal women over the age of 50 years, who had participated in a study of the effect of ERT on knee cartilage in 1998, were invited to have a second examination two years later. ${ }^{12}$ At the time of the initial study, all had been either current, long term users of ERT (five or more years of equivalent dose ERT, by oral, intramuscular, or transdermal route) or non-users of ERT (controls), matched for age $( \pm 5$ years) and years since menopause (as defined by the subjects, \pm 5 years). Subjects had been recruited through the Jean Hailes Centre (a women's health clinic), private consulting clinics, and advertisements in the local media. The study was approved by the ethics committee of the Alfred and Caulfield Hospitals in Melbourne, Australia. All subjects provided informed consent.

The exclusion criteria were: inflammatory arthritis; previous knee joint replacement; malignancy; fracture in the last 10 years; contraindication to magnetic resonance imaging (MRI) (for example, pacemaker, cerebral aneurysm clip, cochlear implant, presence of shrapnel in strategic locations, metal in the eye, or claustrophobia); inability to walk 50 feet $(15.25 \mathrm{~m})$ without the use of a walking aid; 
hemiparesis of either lower limb; and planned total knee replacement.

At baseline, subjects completed a questionnaire to obtain information about demographic data, reproductive and menopausal history, current physical activity, ${ }^{13}$ type and duration of ERT, and smoking history (ever $v$ never smoked). Weight was measured to the nearest $0.1 \mathrm{~kg}$ (shoes and bulky clothing removed) using a single set of electronic scales. Height was measured to the nearest $0.1 \mathrm{~cm}$ (shoes removed) using a stadiometer. BMI $\left(\mathrm{kg} / \mathrm{m}^{2}\right)$ was calculated. Function and pain were assessed by the short form 36 item health survey (SF-36) and the knee specific Western Ontario and McMaster Universities osteoarthritis index (WOMAC). ${ }^{14}{ }^{15} \mathrm{At}$ follow up, information was collected regarding change in ERT status and change in weight.

At baseline, each asymptomatic subject had a weight bearing antero-posterior tibiofemoral radiograph, taken in full extension, of the dominant knee. These radiographs were independently scored in duplicate by a trained observer who used a published atlas to classify disease in the tibiofemoral joint. The radiological features of tibiofemoral osteoarthritis were graded in the medial and lateral compartments on a four point scale (0-3) for individual features of femoral and tibial osteophytes and joint space narrowing. ${ }^{16}$ In the case of disagreement between observers, the films were reviewed with a second independent observer.

Each subject had MRI of the dominant knee, in full extension, at baseline and approximately two years later. The dominant knee was defined as the lower limb from which the subject stepped off when walking. Knee cartilage volume was determined by image processing on an independent workstation. ${ }^{10}{ }^{17}$ Knees were imaged in the sagittal plane on the same $1.5 \mathrm{~T}$ whole body magnetic resonance unit (Signa Advantage HiSpeed, GE Medical Systems, Milwaukee, Wisconsin, USA) using a commercial receive-only extremity coil, as described previously. ${ }^{11}$ Each subject's baseline and follow up MR images were scored within a two week period, unpaired and blinded to subject identification and timing of the MRI. A trained observer read each MRI twice. The two scores (duplicates) for each scan were compared. If the duplicate results were within $\pm 20 \%$, the average of these was used for the volume at the time of the scan. If agreement between the duplicates was outside this range, the MRI was scored again, twice, until the independent measures were within $\pm 20 \%$ and the average used, as previously described. ${ }^{11}$ The coefficients of variation (CV) for the measurement of medial and lateral cartilage volume measures were $3.2 \%$ and $2.7 \%$ (using root mean square CV\% of the results of 10 scans). Total tibial cartilage volume was calculated from the sum of medial and lateral tibial cartilage volume.

Medial and lateral tibial plateau areas were determined by creating an isotropic volume from the input images which were reformatted in the axial plane. Areas were directly measured from these images. CVs for the medial and lateral tibial plateau areas were $2.3 \%$ and $2.4 \%$, respectively. ${ }^{11}$ We did not assume that bone would be stable over time, so the average of the areas taken at baseline and at the two year follow up was used, as bone area was employed as a marker for body size in our analysis.

\section{Statistics}

Descriptive statistics for characteristics of the subjects were tabulated. Independent $t$ tests were used for comparison of means between ERT users and controls. Fisher's exact test was used to compare nominal characteristics between the groups. Annual change in cartilage volume was calculated by:

(Initial knee cartilage volume - follow up knee cartilage volume)

Time between MRI scans

Annual percentage average change in cartilage volume was calculated by:

(Initial knee cartilage volume
- follow up knee cartilage volume)
$\begin{gathered}\text { (Initial knee cartilage volume }) \times \\ \text { (time between MRI scans) }\end{gathered}$

These were the principal outcome measures. Multiple linear regression models were used to explore the possible associations between potential risk factors and change in cartilage volume, including age, height, weight, BMI, WOMAC scores (pain, stiffness, function), bone size, and ERT use. All regression models included cartilage volume at baseline as a covariate to account for any regression to the mean artefact in the changes over time. We explored the association between initial cartilage volume and changes in tibial cartilage volume, adjusted for regression to the mean, using the method of Chuang-Stein and Tong. ${ }^{18}$ All analyses were done using the SPSS statistical package (version 10.0.5, SPSS Inc, Cary, North Carolina, USA). Results are presented as mean $(\mathrm{SD})$.

\section{RESULTS}

Eighty one women were studied at baseline and 57 completed the study $(70 \%)$. Of the 24 subjects who failed to complete the study, 10 could not be contacted, eight declined to be followed for non-specific reasons, four had ill health or metal implants since baseline, and two moved interstate. However, there were no statistically significant differences in any baseline variable between the subjects who were lost to follow up and those who completed the study, including oestrogen use.

The demographic features of the study participants who completed the study are presented in table 1 . When the 24 non-participants are compared with the 57 who participated in the study, no significant differences were observed for the following characteristics: age (mean 56.4 years, $p=0.54$ for difference), BMI (mean $26.9 \mathrm{~kg} / \mathrm{m}^{2}, \mathrm{p}=0.48$ for difference), initial medial cartilage volume (mean $1.53 \mathrm{ml}, \mathrm{p}=0.95$ for difference), initial lateral cartilage volume (mean $2.12 \mathrm{ml}$, $\mathrm{p}=0.17$ for difference), medial tibial plateau area (mean $1672 \mu \mathrm{m}^{2}, \mathrm{p}=0.90$ for difference), and lateral tibial plateau area (mean $1046 \mu \mathrm{m}^{2}, \mathrm{p}=0.67$ for difference).

Among subjects who completed the study, there were no significant differences at baseline between the group that was originally on ERT and the control group, although the control group had slightly more radiographic features of osteoarthritis than the ERT group (table 1). Of the 29 women initially taking ERT ( 17 by mouth, eight transdermally, and three by subcutaneous implant, including 15 taking combined treatment with progesterone at baseline), eight had ceased during the two years of the study. Of the 28 women who entered the study never having been exposed to ERT (control group), three began this treatment over the course of the study.

The mean reduction in "total" tibial cartilage volume per year was $0.09 \mathrm{ml}$ (table 2). When expressed as annual percentage change in cartilage volume, the mean reduction in cartilage volume was $2.4 \%$ of total tibial cartilage per year (95\% confidence interval (CI), $1.5 \%$ to $3.2 \%$ ) (table 2 , fig 1 ). The mean reductions in cartilage volume from the medial and 
Table 1 Baseline clinical characteristics of subjects

\begin{tabular}{|c|c|c|c|c|}
\hline & $\begin{array}{l}\text { Combined (ERT and } \\
\text { controls), } n=57\end{array}$ & ERT, $n=29$ & Controls, $n=28$ & p Value \\
\hline Age (years) & $57(5.9)$ & $58(6.0)$ & $56(5.8)$ & 0.20 \\
\hline Height $(\mathrm{m})$ & $1.64(0.07)$ & $1.65(0.08)$ & $1.63(0.07)$ & 0.57 \\
\hline Weight (kg) & $70.0(13.6)$ & $69.0(11.0)$ & $70.9(15.9)$ & 0.60 \\
\hline BMI $\left(\mathrm{kg} / \mathrm{m}^{2}\right)$ & $26(5.1)$ & $25.5(4.0)$ & $26.6(6.0)$ & 0.42 \\
\hline WOMAC, pain dimension & $2.1(3.1)$ & $2.3(3.6)$ & $1.9(2.5)$ & 0.62 \\
\hline Physical function (SF-36) & $51.2(6.7)$ & $50.5(7.6)$ & $52.0(5.7)$ & 0.39 \\
\hline Mental health function (SF-36) & $51.1(8.0)$ & $50.1(8.2)$ & $52.1(7.8)$ & 0.35 \\
\hline \multicolumn{4}{|l|}{ Radiological osteoarthritis* } & 0.73 \\
\hline \multicolumn{5}{|l|}{ Maximum grade of osteophyte } \\
\hline 0 & 53 & 29 & 24 & 0.05 \\
\hline 1 & 3 & - & 3 & \\
\hline 2 & 1 & - & 1 & \\
\hline \multicolumn{5}{|l|}{$\begin{array}{l}\text { Maximum grade of joint space } \\
\text { narrowing }\end{array}$} \\
\hline 0 & 45 & 25 & 20 & 0.17 \\
\hline 1 & 12 & 4 & 8 & \\
\hline Medial tibial cartilage volume (ml) & $1.53(0.31)$ & $1.56(0.29)$ & $1.49(0.33)$ & 0.41 \\
\hline Lateral tibial cartilage volume (ml) & $1.99(0.38)$ & $2.00(0.37)$ & $1.97(0.40)$ & 0.76 \\
\hline Medial tibial area $\left(\mu \mathrm{m}^{2}\right)$ & $1670(200)$ & $1660(183)$ & $1673(230)$ & 0.80 \\
\hline Lateral tibial area $\left(\mu \mathrm{m}^{2}\right)$ & $1060(140)$ & $1044(142)$ & $1074(132)$ & 0.42 \\
\hline Time between scans (years) & $2.5(0.16)$ & $2.5(0.15)$ & $2.5(0.16)$ & 0.32 \\
\hline \multicolumn{5}{|c|}{$\begin{array}{l}\text { All values are mean (SD). } \\
\text { *Values are the number of subjects in each grade. Fisher's exact test was used to test for differences between } \\
\text { groups. } \\
\text { BMI, body mass index; ERT, oestrogen replacement therapy; SF-36, short form } 36 \text { item general health } \\
\text { questionnaire; WOMAC, Western Ontario and Mc Master Universities osteoarthritis index. }\end{array}$} \\
\hline
\end{tabular}

lateral tibial cartilages were $0.04 \mathrm{ml}$ and $0.05 \mathrm{ml}$, respectively (table 2). Annual average percentage reduction in cartilage volume was similar in the medial and lateral tibial cartilages. The medial cartilage was reduced, on average, by $2.4 \%$ per year ( $1.4 \%$ to $3.3 \%$ ) while the lateral cartilage was reduced, on average, by $2.3 \%$ per year ( $1.2 \%$ to $3.4 \%$ ) (table 2 ).

Change in cartilage volume in the ERT group was compared with that in the control group. No significant differences in the rate of reduction of cartilage volume were demonstrated between the two groups, either in univariate analysis or after adjustment for potential confounding variables (table 3 ). Results were similar when this analysis was repeated using only those subjects who completed the study and remained either ERT users or ERT naïve.

Possible associations between potential risk factors and change in cartilage in healthy postmenopausal women were explored. Univariate and multivariate analysis showed no statistically significant associations with change in cartilage volume in either tibial cartilage (table 4 ). Similar exploratory analyses indicated that change in weight during the period of the study, baseline pain, and baseline exercise level had no significant association with change in the medial or lateral tibial cartilage (results not shown). The association between initial cartilage volume and adjusted changes in lateral tibial cartilage was $-0.03 \mathrm{ml}(-0.09$ to 0.02$)$. This suggests an increased reduction in cartilage volume of $0.03 \mathrm{ml}$ for every increase of $1 \mathrm{ml}$ in initial volume. A similar association was found for medial and total tibial cartilage volume. Analyses were repeated excluding those subjects with radiographic features of osteoarthritis, and the conclusions were unchanged. There was a weak but significant correlation between annual percentage change and reduction in volume in the medial and lateral tibial cartilages (medial percentage loss and lateral percentage loss, correlation coefficient $r=0.30, \mathrm{p}=0.02$; medial volume loss and lateral volume loss, correlation coefficient $r=0.30, \mathrm{p}=0.02$ ).

\section{DISCUSSION}

We found that in a group of 57 healthy postmenopausal women, tibial cartilage was lost over two years at an average rate of $\sim 2.5 \%$ per year. While these data suggest a reduction in cartilage volume over time in such women, based on the 95\% confidence intervals, the average annual reduction may be as low as $1.5 \%$ a year or even as high as $3.4 \%$. We found no effect of ERT on reduction in cartilage volume over two years.

Although the incidence of osteoarthritis in women increases rapidly at about the ages 40 to 50 years, around the menopause, no longitudinal data are available about healthy joints in postmenopausal women, or in healthy adults. $^{3}$ The only available data relate to incidence of radiographic osteoarthritis. In one study, the three year incidence of radiographic knee osteoarthritis (Kellgren

Table 2 Annual average change in tibial cartilage

\begin{tabular}{|c|c|c|c|c|}
\hline & $\begin{array}{l}\text { Annual loss of } \\
\text { cartilage } \\
\text { volume (ml) }\end{array}$ & $\begin{array}{l}95 \% \mathrm{Cl} \text { for mean loss of } \\
\text { cartilage volume }(\mathrm{ml})\end{array}$ & $\begin{array}{l}\text { Annual loss of } \\
\text { cartilage volume (\%) }\end{array}$ & $\begin{array}{l}95 \% \mathrm{Cl} \text { for average } \\
\% \text { change }\end{array}$ \\
\hline Total tibial cartilage $†$ & $0.09(0.11)$ & 0.06 to 0.12 & $2.4(3.2)$ & 1.5 to 3.2 \\
\hline Medial tibial cartilage & $0.04(0.06)$ & 0.03 to 0.05 & $2.4(3.6)$ & 1.4 to 3.3 \\
\hline Lateral tibial cartilage & $0.05(0.08)$ & 0.03 to 0.07 & $2.3(4.2)$ & 1.2 to 3.4 \\
\hline $\begin{array}{l}\text { Values are mean (SD). } \\
\text { †Total = medial+lateral. } \\
\mathrm{Cl} \text {, confidence interval. }\end{array}$ & & & & \\
\hline
\end{tabular}




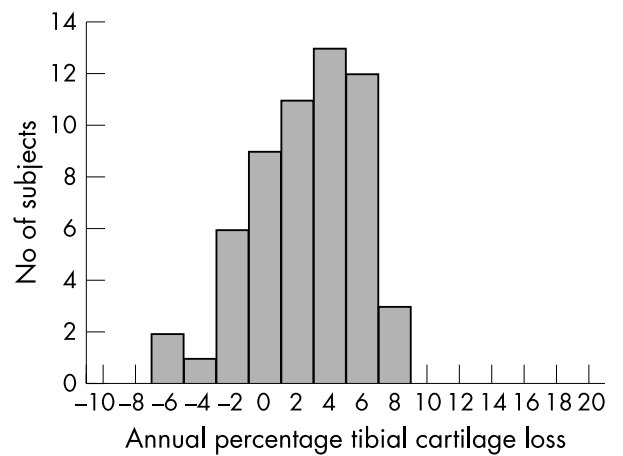

Figure 1 Individual annual percentage rate of tibial cartilage loss for healthy postmenopausal women. The $y$ axis represents the number of subjects with a given percentage of tibial cartilage loss. The mean (SD) annual rate of cartilage loss was $2.4(3.2) \%(n=57)$.

Lawrence) in a group of postmenopausal women was shown to be approximately $1 \% .{ }^{19}$ Cross sectional data suggest that older women (aged 50 to 78 years) have thinner total knee cartilage than younger women (aged 20 to 30 years), implying previous loss in the elderly women. ${ }^{9}$ The only published longitudinal data regarding average change in cartilage in a healthy population come from a small series $(\mathrm{n}=13) .^{20}$ This showed an average annual cartilage gain. However, this series included both men $(n=5)$ and women $(\mathrm{n}=8)$, and involved a younger population (mean (SD) age, 49 (18) years) than the one we describe here. ${ }^{20}$ However, the 95\% confidence interval for average annual change in cartilage (gain of $6.7 \%$ a year, loss of $2.3 \%$ a year) overlapped those of the present study in healthy adults. In contrast to these studies in healthy subjects, subjects with osteoarthritis lose tibial cartilage at an average annual rate of 5.3\% (4.4\% to $6.2 \%)$, approximately double the rate observed in these healthy women. ${ }^{11}$

We showed in a healthy, well characterised population of postmenopausal women that the reduction in cartilage volume lies between $1.5 \%$ and $3.4 \%$ a year, with the average change being $2.4 \%$ of total tibial cartilage per year. At an individual level, the minimum detectable change that can be distinguished from measurement error (at a 5\% level of significance) is 2.8 multiplied by the coefficient of variation for an individual volume measurement-that is, approximately $8 \%$ for total tibial cartilage volume in this study. ${ }^{21}$ Thus no individual had a change in cartilage volume that was outside the limits of measurement error at the individual level. ${ }^{21}$ Despite this, it may be that some subjects truly gained cartilage over the course of the study, or that swelling occurred in existing cartilage. Only improvements in measurement technique-including developing the ability to
Table 4 Associations between potential risk factors and change in tibial cartilage volume $\left(\mathrm{mm}^{3}\right)$ in healthy women (ERT and controls, $n=57$ )

\begin{tabular}{|c|c|c|c|}
\hline & $\begin{array}{l}\text { Univariate } \\
\text { regression } \\
\text { coefficient }\end{array}$ & $\begin{array}{l}\text { Multivariate } \\
\text { regression } \\
\text { coefficient }^{*}\end{array}$ & $95 \% \mathrm{Cl}$ \\
\hline \multicolumn{4}{|l|}{ Medial cartilage } \\
\hline Age† (years) & 0.06 & 0.39 & -2.0 to 2.8 \\
\hline Height‡ (m) & 107 & - & - \\
\hline Weight\$ (kg) & 0.20 & - & - \\
\hline BMI $\left(\mathrm{kg} / \mathrm{m}^{2}\right)$ & -0.13 & -0.53 & -3.4 to 2.3 \\
\hline ERT use ${ }^{\star \star}$ & 20.5 & 12.7 & -15.5 to 40.8 \\
\hline Bone areatt $\left(\mu \mathrm{m}^{2}\right)$ & 0.04 & -0.02 & -0.10 to 0.06 \\
\hline \multicolumn{4}{|l|}{ Lateral cartilage } \\
\hline Age† (years) & -2.7 & -1.04 & -4.7 to 2.6 \\
\hline Height‡ $(\mathrm{m})$ & 26.4 & - & - \\
\hline Weight\$ (kg) & -0.04 & - & - \\
\hline BMI $\left(\mathrm{kg} / \mathrm{m}^{2}\right)$ & -0.13 & 0.70 & -3.51 to 4.90 \\
\hline ERT use ${ }^{* \star}$ & -3.8 & -8.5 & -49.5 to 32.6 \\
\hline Bone areatt $\left(\mu \mathrm{m}^{2}\right)$ & -0.05 & -0.16 & -0.32 to 0.01 \\
\hline \multicolumn{4}{|l|}{ Total cartilage } \\
\hline Aget (years) & -2.7 & -0.81 & -5.7 to 4.1 \\
\hline Heightł $(\mathrm{m})$ & 133.7 & - & - \\
\hline Weight§ (kg) & 0.16 & - & - \\
\hline BMI $\left(\mathrm{kg} / \mathrm{m}^{2}\right)$ & -0.26 & 0.12 & -5.8 to 6.0 \\
\hline ERT use ${ }^{* *}$ & 16.7 & 6.4 & -49.9 to 62.8 \\
\hline Bone areatt $\left(\mu \mathrm{m}^{2}\right)$ & 0.02 & -0.07 & -0.18 to 0.04 \\
\hline
\end{tabular}

*Initial cartilage volume included in the regression model, with age, body mass index, hormone replacement therapy use, and bone area. † Volume change $\left(\mathrm{mm}^{3}\right)$ per year increase in age. $\ddagger$ Volume change $\left(\mathrm{mm}^{3}\right)$ per $\mathrm{cm}$ increase in height. $\S$ Volume change $\left(\mathrm{mm}^{3}\right)$ per $\mathrm{kg}$ increase in weight. - Volume change $\left(\mathrm{mm}^{3}\right)$ per unit increase in BMI.

**Controls $v$ women on ERT.

††Volume change $\left(\mathrm{mm}^{3}\right)$ per $1 \mu \mathrm{m}^{2}$ increase in bone area. $\mathrm{BMI}$, body mass index; $\mathrm{Cl}$, confidence interval; ERT, oestrogen replacement therapy.

identify normal cartilage-will answer this question. Indeed, it is also accepted that this estimate of the minimum detectable difference may underestimate the confidence intervals of the measurement. ${ }^{21}$

There is evidence to suggest that ERT may be protective of incident osteoarthritis in postmenopausal women. ${ }^{322}$ We have shown that long term current users of ERT have more tibial cartilage than non-users. ${ }^{12}$ However, our study showed no effect of ERT on change in cartilage volume over two years. There are various possible explanations for this finding which could reconcile the discrepancy between baseline and follow up studies in the effect of ERT on cartilage volume. It may be that our sample size was too small to show an effect. However, the direction and magnitude of change in cartilage volume was similar in both groups. Alternatively, it may be that cartilage volume is lost most rapidly early in the

Table 3 Annual average change in cartilage in ERT subjects $v$ controls

\begin{tabular}{|c|c|c|c|c|c|c|}
\hline & ERT $(n=29)$ & Control $(n=28)$ & Difference $(95 \% \mathrm{Cl})$ & p Value & Adjusted difference $(95 \% \mathrm{Cl}) \dagger$ & p Value \\
\hline \multicolumn{7}{|l|}{ Amount lost (ml) } \\
\hline Total tibial cartilage & $0.10(0.09)$ & $0.08(0.13)$ & $0.02(-0.04$ to 0.08$)$ & 0.58 & $0.006(-0.05$ to 0.06$)$ & 0.82 \\
\hline Medial tibial cartilage & $0.05(0.05)$ & $0.03(0.06)$ & $0.02(-0.01$ to 0.05$)$ & 0.17 & $0.013(-0.02$ to 0.04$)$ & 0.37 \\
\hline Lateral tibial cartilage & $0.05(0.06)$ & $0.05(0.10)$ & $-0.004(-0.05$ to 0.04$)$ & 0.86 & $0.008(-0.05$ to 0.03$)$ & 0.68 \\
\hline \multicolumn{7}{|l|}{ Reduction (\%) } \\
\hline Total tibial cartilage & $2.7(2.5)$ & $2.1(3.8)$ & $0.5(-1.2$ to 2.3$)$ & 0.53 & $0.3(-1.4$ to 2.0$)$ & 0.73 \\
\hline Medial tibial cartilage & $3.1(3.4)$ & $1.6(3.8)$ & $1.5(-0.5$ to 3.4$)$ & 0.13 & $1.0(-0.9$ to 3.0$)$ & 0.29 \\
\hline Lateral tibial cartilage & $2.2(2.9)$ & $2.4(5.3)$ & $-0.1(-2.5$ to 2.5$)$ & 0.90 & $0.4(-2.7$ to 1.8$)$ & 0.72 \\
\hline
\end{tabular}

Results are mean (SD) or mean ( $95 \%$ confidence interval).

Differences and $p$ values compare results in the ERT group with the control group

†Adjusted for age, body mass index, bone area and initial cartilage volume.

$\mathrm{Cl}$, confidence interval; ERT, oestrogen replacement therapy. 
menopausal period, so that use of ERT at that time has an apparently stronger effect than when used well after the menopause. At baseline, all women who had been on ERT had been on it for at least five years, usually earlier in the menopausal period, accounting for the higher baseline volumes seen in the initial study. Later on, following the menopause, the rate of reduction in cartilage volume may slow so that the observed effect of oestrogen is less. Alternatively, it may be that following withdrawal of ERT, a rapid reduction in cartilage volume occurs, similar to the effect seen on bone mineral density when ERT is withdrawn, and this rapid loss overshadows any protective effect. It is also possible that ERT does not act directly on articular cartilage but indirectly on other structures such as muscle and bone, with an indirect effect on cartilage early in the menopause.

The limitations of our study relate predominantly to generalisability and measurement issues. Subjects were recruited on the basis of ERT use, and excluded if they had significant knee pathology or symptoms. This is likely to have selected a healthier group of women and may possibly underestimate the average rate of reduction in cartilage volume in postmenopausal women. These results are generalisable only to asymptomatic subjects, as those with pain were excluded. We may have overestimated the reduction in cartilage volume in these women, as we did not exclude asymptomatic subjects with radiographic signs suggestive of osteoarthritis (osteophytes and joint space narrowing). However, we repeated the analysis excluding these subjects and found no differences. It could also be the case that the women who were lost to follow up lost cartilage at a different rate from those who completed the study. However, these women did not differ in any measurable way from those who completed the study, and did not withdraw from the study for reasons related to their knees. Our study has small numbers and so has limited ability to detect significant effects on change in cartilage volume. It is also possible that changes in the type of ERT used may vary. However, for inclusion in the ERT group subjects had to be on equivalent doses of ERT, whether by oral, transdermal, or intramuscular route.

The measurement of cartilage volume is dependent on the clarity of the contrast between cartilage and the adjacent tissues and the minimisation of positioning differences and partial volume averaging error. To optimise the images and improve resolution, we use fine slices of $1.5 \mathrm{~mm}$ width and a matrix of $512 \times 192$ pixels, resulting in an in-plane resolution of $0.31 \times 0.83 \mathrm{~mm}$. Our method has been validated against cadaveric and postoperative specimens using water displacement, and has excellent reproducibility $(2-3 \%) .{ }^{17}$ We measured tibial cartilage only in these particular subjects, having previously shown that tibial and femoral cartilage volumes $^{23}$ and change in tibial and femoral cartilage are

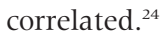

The factors affecting cartilage growth and possible decline throughout life are poorly understood. Although children with higher levels of physical activity and normal BMI have more cartilage than those with low level physical activity and high BMI, the age at which cartilage gain ceases is unknown. ${ }^{10}$ Indeed, whether it remains stable throughout adult life is unknown. It is possible that cartilage volume is relatively stable before the age of 40 to 50 , and is lost more rapidly thereafter, or lost steadily throughout adult life, unless a trigger for the development of osteoarthritis occurs. Hormonal change in the perimenopausal period may act as such a trigger. Cartilage volume may diminish more rapidly in the perimenopausal period, in a fashion similar to the reduction in bone mineral content. ${ }^{25}$ This may in part explain the increase in the incidence of osteoarthritis in the perimenopausal period. In the same period, symptoms of osteoarthritis are common. We have recently shown that loss of tibial cartilage is associated with increased pain, stiffness, and loss of function related to the knee. ${ }^{26}$ Thus cartilage change during the menopause in non-osteoarthritic women may provide one potential mechanism for the onset of osteoarthritis in postmenopausal women. With current MRI technology, it is not possible to determine the quality of articular cartilage and what components of its structure contribute to the reduction in cartilage volume. However, developments in technology may make this possible in the future.

In postmenopausal women the average rate of reduction of tibial cartilage volume lies between $1.5 \%$ and $3.4 \%$ a year. Further studies will be required to determine whether similar changes occur in healthy men, and whether change in cartilage is steady through adult life, or whether there is a rapid decline in women in the menopausal period.

\section{ACKNOWLEDGEMENTS}

This study was supported by the Shepherd Foundation. AEW is the recipient of a National Health and Medical Research Council scholarship with additional funds from the Alfred Research Trusts.

\section{Authors' affiliations}

A E Wluka, R Wolfe, F M Cicuttini, Department of Epidemiology and Preventive Medicine, Monash University Medical School, Alfred Hospital, Melbourne, Australia

S R Davis, Jean Hailes Foundation, Clayton, Victoria, Australia

S Stuckey, MRI Unit, Radiology Department, Alfred Hospital, Melbourne

\section{REFERENCES}

1 Slemenda C, Heilman DK, Brandt KD, Katz BP, Mazzuca SA, Braunstein EM, et al. Reduced quadriceps strength relative to body weight: a risk factor for knee osteoarthritis in women? Arthritis Rheum 1998;41:1951-9.

2 Cooper C, Snow S, McAlindon TE, Kellingray S, Stuart B, Coggon D, et al. Risk factors for the incidence and progression of radiographic knee osteoarthritis. Arthritis Rheum 2000;43:995-1000.

3 Nevitt MC, Felson DT. Sex hormones and the risk of osteoarthritis in women: epidemiological evidence. Ann Rheum Dis 1996;55:673-6.

4 Bayliss MT, Ali SY. Age-related changes in the composition and structure of human articular-cartilage proteoglycans. Biochem J 1978; 176:683-93.

5 Glant T, Mikecz K, Roughley PJ, Buzas E, Poole AR. Age-related changes in protein-related epitopes of human articular-cartilage proteoglycans. Biochem $J$ 1986:236:71-5

6 Roughley PJ, Mort JS. Ageing and the aggregating proteoglycans of human articular cartilage. Clin Sci (Colch) 1986;71:337-44.

7 Stanescu V, Chaminade F, Muriel MP. Age-related changes in small proteoglycans of low buoyant density of human articular cartilage. Connect Tissue Res 1988; 17:239-52.

8 Verziil N, DeGroot J, Ben ZC, Brau-Benjamin O, Maroudas A, Bank RA, et al. Crosslinking by advanced glycation end products increases the stiffness of the collagen network in human articular cartilage a possible mechanism through which age is a risk factor for osteoarthritis. Arthritis Rheum 2002;46:114-23.

9 Hudelmaier M, Glaser C, Hohe J, Englmeier KH, Reiser M, Putz R, et al. Agerelated changes in the morphology and deformational behavior of knee joint cartilage. Arthritis Rheum 2001;44:2556-61.

10 Jones G, Glisson M, Hynes K, Cicuttini F. Sex and site differences in cartilage development: a possible explanation for variations in knee osteoarthritis in later life. Arthritis Rheum 2000;43:2543-9.

11 Wluka AE, Stuckey S, Snaddon J, Cicuttini FM. The determinants of change in tibial cartilage volume in osteoarthritic knees. Arthritis Rheum 2002;46:2065-72.

12 Wluka AE, Davis SR, Bailey M, Stuckey SL, Cicuttini FM. Users of oestrogen replacement therapy have more knee cartilage than non-users. Ann Rheum Dis 2001;60:332-6.

13 Spector TD, Harris PA, Hart DJ, Cicuttini FM, Nandra D, Etherington J, et al. Risk of osteoarthritis associated with long-term weight-bearing sports: a radiologic survey of the hips and knees in female ex-athletes and population controls. Arthritis Rheum 1996;39:988-95.

14 Ware JEJ, Snow KK, Kosinski M, Gandek B. SF-36 health survey: manual and interpretation guide. Boston: The Health Institute, New England Medical Center, 1993.

15 Bellamy N, Buchannan WW, Goldsmith CH, Campbell J, Stitt LW. Validation study of WOMAC: a health status instrument for measuring clinically important patient relevant outcomes to antirheumatic drug therapy in patients with osteoarthritis of the hip or knee. J Rheumatol 1988; 15:1833-40. 
16 Altman RD, Hochberg M, Murphy WA, Wolfe F, Lequesne M. Atlas of individual radiographic features in osteoarthritis. Osteoarthritis Cartilage 1995;3(suppl A):3-70.

17 Cicuttini F, Forbes A, Morris K, Darling S, Bailey M, Stuckey S. Gender differences in knee cartilage volume as measured by magnetic resonance imaging. Osteoarthritis Cartilage 1999;7:265-71.

18 Chuang-Stein C, Tong DM. The impact and implication of regression to the mean on the design and analysis of medical investigations. Stat Methods Med Res 1997;6: 115-28.

19 Sowers M, Lachance L, Jamadar D, Hochberg MC, Hollis B, Crutchfield M, et al. The associations of bone mineral density and bone turnover markers with osteoarthritis of the hand and knee in pre- and perimenopausal women [see comments]. Arthritis Rheum 1999:42:483-9.

20 Cicuttini FM, Forbes A, Yuanyuan W, Rush G, Stuckey SL. Rate of knee cartilage loss after partial meniscectomy. J Rheumatol 2002;29:1954-6.
21 Cummings SR, Black D. Should perimenopausal women be screened for osteoporosis? Ann Intern Med 1986; 104:817-23.

22 Wluka AE, Cicuttini FM, Spector TD. Menopause, oestrogens and arthritis. Maturitas 2000;35:183-99.

23 Cicuttini FM, Wluka AE, Stuckey SL. Tibial and femoral cartilage changes in knee osteoarthritis. Ann Rheum Dis 2001;60:977-80.

24 Cicuttini FM, Wluka AE, Wang Y, Stuckey SL. Longitudinal study of changes in tibial and femoral cartilage in knee osteoarthritis. Arthritis Rheum

25 Melton LI, Atkinson EJ, O'Connor MK, O'Fallon WM, Riggs BL. Determinants of bone loss from the femoral neck in women of different ages. J Bone Miner Res 2000;15:24-31.

26 Wluka AE, Wolfe R, SL S, Cicuttini FM. How does tibial cartilage volume relate to symptoms in subjects with knee osteoarthritis? Ann Rheum Dis (in press). 